\title{
ADUBAÇÃO N-P-K E O DESENVOLVIMENTO, PRODUTIVIDADE E QUALIDADE DOS FRUTOS DO ABACAXI 'GOLD' (MD-2) ${ }^{(1)}$
}

\author{
André Guarçoni M. ${ }^{(2)}$ \& José Aires Ventura ${ }^{(3)}$
}

\begin{abstract}
RESUMO
O abacaxi Gold (MD-2) tem atraído interesse no Brasil, visando à exportação. No entanto, há poucas informações científicas no País sobre o manejo nutricional dessa cultivar. $O$ presente trabalho teve por objetivo determinar o efeito da adubação com $\mathrm{N}$, P e K no estado nutricional da planta, no desenvolvimento, na produtividade e na qualidade dos frutos do abacaxi MD-2. Foram avaliados os efeitos de cinco doses de $\mathrm{N}$, cinco de $\mathrm{P}_{2} \mathrm{O}_{5}$ e cinco de $\mathrm{K}_{2} \mathrm{O}$ sobre as características de crescimento da folha $\mathrm{D}$ e do fruto, bem como sobre os teores de $\mathrm{N}, \mathrm{P}$ e $\mathrm{K}$ das folhas $\mathrm{D}$ e as características de qualidade do fruto. Concluiu-se que as maiores produtividade e massa de fruto foram obtidas com a aplicação de $650,6 \mathrm{~kg} \mathrm{ha}^{-1} \mathrm{de}$ $\mathrm{N}$ e $735,9 \mathrm{~kg} \mathrm{ha}^{-1}$ de $\mathrm{K}_{2} \mathrm{O}$, correspondendo a 12,7 e $14,4 \mathrm{~g} / \mathrm{planta}$ de $\mathrm{N}$ e $\mathrm{K}_{2} \mathrm{O}$, respectivamente. Nesse caso, a indução floral deve ser recomendada quando a folha $D$ apresentar comprimento $\geq 75,5 \mathrm{~cm}$. Os valores das características de qualidade do fruto diminuíram com a aplicação de $\mathrm{N}$ e aumentaram com a adição

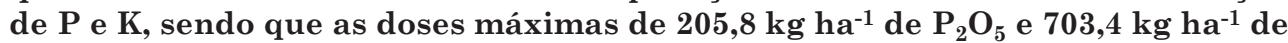
$\mathrm{K}_{2} \mathrm{O}$ corresponderam a 4,01 e 13,7 g/planta de $\mathrm{N}$ e $\mathrm{K}_{2} \mathrm{O}$, respectivamente.
\end{abstract}

Termos de indexação: Ananas comosus var. comosus, fertilização, macronutrientes, crescimento.

\section{SUMMARY: NITROGEN, PANDK FERTILIZATIONANDTHE DEVELOPMENT, YIELD AND FRUIT QUALITY OF PINEAPPLE 'GOLD'(MD-2)}

The cultivar Gold (MD-2) has attracted interest in Brazil, with a view to fresh pineapple export. However, little scientific information is available on the nutritional management of this cultivar in the country. This study aimed to determine the effect of $N$, P and Kfertilization

\footnotetext{
(1) Recebido para publicação em 29 de setembro de 2010 e aprovado em 24 de maio de 2011.

(2) Incaper, CRDR - Centro Serrano, BR 262, km 94, Fazenda do Estado, CEP 29375-000 Venda Nova do Imigrante (ES). E-mail: guarconi@incaper.es.gov.br

${ }^{(3)}$ Incaper, Sede, Rua Afonso Sarlo 160, Bento Ferreira, CEP 29052-010, Vitória (ES). E-mail: ventura@incaper.es.gov.br
} 


\begin{abstract}
on the nutritional status, development, yield and fruit quality of MD-2 pineapple plants. Five doses of $\mathrm{N} ; \mathrm{P}_{2} \mathrm{O}_{5}$ and $\mathrm{K}_{2} \mathrm{O}$, were studied and the leaf ' $D$ ' development, the $\mathrm{N}$, $P$ and $K$ levels, and fruit development and quality were evaluated. The yield and fruit weight were highest after the application of $650.6 \mathrm{~kg} \mathrm{ha}^{-1} \mathrm{~N}$ and $735.9 \mathrm{~kg} \mathrm{ha}^{-1} \mathrm{~K}_{2} \mathrm{O}$, corresponding to 12.7 and $14.4 \mathrm{~g} /$ plant, respectively; in this case, flowering should be induced when the 'D'leaf length is $\geq 75.5 \mathrm{~cm}$; the fruit quality was reduced with application of $N$, but increased with the addition of $P$ and $K$, up to a maximum of $205.8 \mathrm{~kg} \mathrm{ha}^{-1} \mathrm{P}_{2} \mathrm{O}_{5}$ and $703.4 \mathrm{~kg} \mathrm{ha}^{-1} \mathrm{~K}_{2} \mathrm{O}$, corresponding to 4.01 and $13.7 \mathrm{~g} /$ plant, respectively.
\end{abstract}

Index terms: Ananas comosus var. comosus, fertilization, macronutrients, plant growth.

\section{INTRODUÇÃO}

O abacaxi Gold (MD-2), híbrido desenvolvido no Havaí e originado do cruzamento dos híbridos PRI 58-1184 e 59-443, apresenta baixo teor de acidez, elevado teor de sólidos solúveis totais, formato uniforme e polpa amarelada (Morgan \& Thompson, 2000; Chan et al., 2002); sendo que vem assumindo papel de destaque no mercado consumidor internacional. Em vista disso, as empresas brasileiras exportadoras de frutas têm mostrado grande interesse por essa cultivar. Entretanto, apenas com a oferta de frutos de excelente qualidade pode-se atingir competitividade internacional.

A utilização de adubos na cultura do abacaxi constitui-se em prática obrigatória nos plantios com fins comerciais, devido ao elevado grau de exigência da planta, o qual varia com a cultivar plantada (Reinhardt et al., 2002). No entanto, poucas são as informações científicas no Brasil sobre o cultivo do MD-2, especialmente as relativas à nutrição das plantas.

Além das exigências nutricionais da planta e da capacidade de suprimento de nutrientes pelo solo, fatores como o nível tecnológico adotado no cultivo, a destinação da produção e a rentabilidade da cultura devem ser considerados para a definição sobre as quantidades de fertilizantes a serem aplicadas (Reinhardt et al., 2002). As variações que podem ocorrer nesse conjunto de fatores, quando se avaliam características de produção de diferentes regiões, evidenciam que as recomendações de adubação devem levar em consideração a abrangência regional.

Acredita-se que a resposta à adubação da cultivar MD-2 seja similar à resposta do abacaxi Smooth Cayenne. Este, por sua vez, apresenta seu desenvolvimento e a qualidade dos frutos altamente influenciados pelos nutrientes $\mathrm{N}$ e $\mathrm{K}$. O primeiro propicia frutos de maior tamanho, mas, em contrapartida, tende a reduzir os teores de sólidos solúveis e a acidez. Já o K tem menor influência no desenvolvimento do fruto do que o $\mathrm{N}$, porém é o nutriente que mais influencia a qualidade do fruto (Teixeira et al., 2002; Spironello et al., 2004). Para a adubação fosfatada, Botrel et al. (1991) e Spironello et al. (2004) observaram pequena resposta ou nula, tanto no desenvolvimento quanto na qualidade do fruto, mesmo em solo com baixos teores de P.
O espaçamento de plantio adotado promove alterações na produtividade e na massa dos frutos. Segundo Santana et al. (2001), para cada acréscimo de 10.000 plantas ha ${ }^{-1}$ da cultivar Smooth Cayenne, o peso médio do fruto diminuiu $102 \mathrm{~g}$ e a produtividade aumentou em 8,27 tha-1. Para a cultivar MD-2, entretanto, Rebolledo et al. (2006) observaram que, para cada acréscimo de 10.000 plantas por hectare, a redução no peso médio do fruto foi de $106 \mathrm{~g}$, porém com incremento na produtividade de $16 \mathrm{t} \mathrm{ha}^{-1}$, aproximadamente. Essas alterações influenciam claramente a demanda por nutrientes. Assim, as doses de nutrientes atualmente aplicadas para o abacaxi MD-2 podem estar aquém da real necessidade da cultura.

O presente trabalho teve por objetivo determinar os efeitos da adubação com N, P e K no estado nutricional à época da indução floral das plantas, no desenvolvimento, na produtividade e na qualidade dos frutos do abacaxi Gold (MD-2), em plantio adensado, na região norte do Estado do Espírito Santo.

\section{MATERIAL E MÉTODOS}

Foi instalado um experimento utilizando o abacaxi cv. Gold (MD-2), na Fazenda Experimental de Sooretama (ES), pertencente ao Instituto Capixaba de Pesquisa, Assistência Técnica e Extensão Rural (Incaper), em área irrigada por aspersão, num Latossolo VermelhoAmarelo distrófico com as seguintes características químicas: $\mathrm{pH}\left(\mathrm{H}_{2} \mathrm{O}\right)=5,3 ; \mathrm{Pe} \mathrm{K}=2,56$ e $81 \mathrm{mg} \mathrm{dm}^{-3}$, respectivamente; $\mathrm{Ca}^{2+}, \mathrm{Mg}^{2+}$ e $\mathrm{Al}^{3+}=0,98,0,47 \mathrm{e}$ $0,31 \mathrm{cmol}_{\mathrm{c}} \mathrm{dm}^{-3}$, respectivamente; $\mathrm{e}$ P-remanescente $=$ $36,89 \mathrm{mg} \mathrm{L}^{-1}$.

A necessidade de calagem foi calculada utilizandose o método da neutralização do alumínio $(\mathrm{Y}=0,82)$ e elevação dos teores de $\left.\mathrm{Ca}^{2+}{\mathrm{e} \mathrm{Mg}^{2+}}^{2+} \mathrm{X}=2\right)$, com aplicação, no final de novembro, de calcário dolomítico até a profundidade de $20 \mathrm{~cm}$. No início de janeiro, foi efetuado o plantio das mudas, em fileiras duplas com espaçamento de $90 \times 40 \times 30 \mathrm{~cm}$, gerando uma densidade de 51.280 plantas ha-1. $^{-1}$.

Os tratamentos foram formados por cinco doses de $\mathrm{N}$, cinco de $\mathrm{P}_{2} \mathrm{O}_{5}$ e cinco de $\mathrm{K}_{2} \mathrm{O}$, combinadas segundo a matriz experimental Plan Puebla III $\left(2^{\mathrm{k}}+2 \mathrm{k}+1\right)$, 
acrescida de um ponto, sendo os limites mínimos e máximos de 0 a 1.000 , 0 a 400 e 0 a $1.000 \mathrm{~kg} \mathrm{ha}^{-1}$ para $\mathrm{N}, \mathrm{P}_{2} \mathrm{O}_{5}$ e $\mathrm{K}_{2} \mathrm{O}$, respectivamente, perfazendo 16 tratamentos. A adubação fosfatada, na forma de superfosfato triplo, foi aplicada de uma só vez no sulco de plantio. $\mathrm{N}$ e $\mathrm{K}$ foram aplicados, em cobertura (aplicação axilar), nos quatro parcelamentos seguintes: no primeiro (12\%), terceiro (18\%), sexto (28\%) e nono ( $42 \%$ da dose) mês após o plantio, na forma de ureia e $\mathrm{KCl}$, respectivamente. No nono mês após o plantio, foi também realizada, como fator mantido constante, uma aplicação foliar de $40 \mathrm{~mL} /$ planta de uma solução contendo Zn, Mn, Fe e B, na concentração de 1,5 g L $\mathrm{L}^{-1}$.

Os tratamentos foram distribuídos em blocos ao acaso com três repetições, gerando um total de 48 parcelas experimentais, sendo estas constituídas por duas fileiras de 14 plantas, considerando úteis as 24 centrais em cada parcela.

A indução floral foi realizada com ethefon (Ethrel $\left.{ }^{\circledR}\right)$ no $11^{\circ}$ mês após o plantio, sendo a colheita finalizada 6,5 meses após a indução floral.

Uma semana antes da indução floral, foram coletadas as folhas $\mathrm{D}$ de 20 plantas úteis de cada parcela. O comprimento dessas folhas foi medido, sendo então separadas em parte basal aclorofilada e parte clorofilada. As amostras foliares foram secas em estufa de circulação forçada até massa constante, determinando-se a massa de matéria seca total das folhas D. As partes basais aclorofiladas foram moídas em moinho tipo Wiley e mineralizadas por via úmida com solução sulfúrica, para determinar os teores totais de $\mathrm{N}$, e nítrico-perclórica, para determinação dos teores totais de $\mathrm{P}$ e $\mathrm{K}$.

Os frutos foram colhidos, sendo determinados o diâmetro dos frutos (DF), sua massa com coroa (MCC) e sem coroa (MSC) e a percentagem da massa da coroa em relação à massa do fruto com coroa $(\% \mathrm{MC}=$ Massa da coroa x 100/MCC). A partir da MCC, foi estimada a produção em t ha-1. Determinou-se, no suco da polpa dos frutos, a acidez total titulável (ATT, em \% ácido cítrico), os teores de sólidos solúveis totais (SST, em ${ }^{\circ}$ Brix) e a relação SST/ATT (ratio).

Após a colheita dos frutos, foram coletadas 15 amostras simples de solo por parcela, as quais foram secas ao ar e homogeneizadas, gerando uma amostra composta por parcela, que foram analisadas quimicamente, sendo determinados os teores de $\mathrm{P}$ e K disponíveis (Mehlich-1).

Funções de resposta, do tipo: $\mathrm{Y}=\mathrm{b}_{0}+\mathrm{b}_{1} \mathrm{~N}+\mathrm{b}_{2} \mathrm{~N}^{2}$ $+\mathrm{b}_{3} \mathrm{P}+\mathrm{b}_{4} \mathrm{P}^{2}+\mathrm{b}_{5} \mathrm{~K}+\mathrm{b}_{6} \mathrm{~K}^{2}+\mathrm{b}_{7} \mathrm{NP}+\mathrm{b}_{8} \mathrm{NK}+\mathrm{b}_{9}$ $\mathrm{PK}$, foram ajustadas para todas as variáveis avaliadas, sendo $\mathrm{Y}$ a variável dependente, $\mathrm{b}_{0} . . . \mathrm{b}_{9}$ os coeficientes da regressão e $\mathrm{N}, \mathrm{P}$ e $\mathrm{K}$ as doses de $\mathrm{N}, \mathrm{P}_{2} \mathrm{O}_{5}$ e $\mathrm{K}_{2} \mathrm{O}$, respectivamente. A significância dos coeficientes $\left(b_{0} \ldots b_{9}\right)$ foi testada, e novas funções de resposta foram ajustadas, considerando apenas os efeitos significativos $(\mathrm{p} \leq 0,10)$. Simultaneamente, foram calculadas as doses e combinações de $\mathrm{N}, \mathrm{P}_{2} \mathrm{O}_{5}$ e $\mathrm{K}_{2} \mathrm{O}$, para obtenção de valores máximos das variáveis dependentes. Foram realizadas análises de correlação do comprimento, da massa, dos teores de N, P e K e das relações entre esses nutrientes, das folhas $\mathrm{D}$, com as características de desenvolvimento e de qualidade dos frutos.

\section{RESULTADOS E DISCUSSÃO}

\section{Comprimento, massa de matéria seca e teor de nutrientes na folha $D$}

Os valores das características de desenvolvimento foliar aumentaram de forma quadrática em decorrência da aplicação de doses crescentes dos nutrientes $\mathrm{N}$ e $\mathrm{K}$ (Quadros 1 e 2). O comprimento máximo da folha $\mathrm{D}$, obtido com 582,2 e $542,6 \mathrm{~kg} \mathrm{ha}^{-1}$ de $\mathrm{Ne} \mathrm{K}_{2} \mathrm{O}$, respectivamente, foi estimado em $76,6 \mathrm{~cm}$, sendo este maior do que o comprimento máximo reportado por Reinhardt et al. (2002) para a cultivar Smooth Cayene $(68 \mathrm{~cm})$. A massa de matéria seca máxima da folha $\mathrm{D}$ foi estimada em $6,84 \mathrm{~g}$, com aplicação de 638,0 e $571,9 \mathrm{~kg} \mathrm{ha}^{-1}$ de $\mathrm{N}$ e $\mathrm{K}_{2} \mathrm{O}$, respectivamente. Bhugaloo (1998), trabalhando com a cv. Queen Victoria, observou efeito positivo de doses crescentes de $\mathrm{N}$ sobre o comprimento da folha $\mathrm{D}$, mas não sobre sua massa.

A adubação com ureia aumentou, de forma linear, os teores foliares de N, porém reduziu os teores foliares de $\mathrm{P}$ e a relação K:N (Quadros 1 e 2). A redução nos teores foliares de $\mathrm{P}$ não é explicada apenas por um possível efeito de diluição, como consequência do alongamento da folha $\mathrm{D}$, gerado principalmente pela adição de $\mathrm{N}$ na forma de ureia (Quadro 2), razão apontada por Spironello et al. (2004) como causa da redução nos teores foliares de P no abacaxi Smooth Cayenne. Nightingale (1942) relata que elevados teores de nitrato no solo podem causar correspondente redução na absorção de $\mathrm{P}$ pelo abacaxi. Considerando que a passagem de uréia a amônio (Longo \& Melo, 2005) e, posteriormente, a nitrato (Rosolem et al., 2003) é rápida em solo com pH corrigido, como no caso do presente estudo, a redução nos teores foliares de $\mathrm{P}$ pode ser, também, consequência de teores mais elevados de nitrato no solo.

A aplicação de superfosfato triplo elevou os teores foliares de $\mathrm{N}$ e de $\mathrm{P}$ de forma linear e os de $\mathrm{K}$ e a relação $\mathrm{K}: \mathrm{N}$ de forma quadrática, sendo a dose máxima de $\mathrm{P}_{2} \mathrm{O}_{5}$, nesses casos, igual a $238 \mathrm{~kg}$ ha-1 ou 4,64 g/planta (Quadros 1 e 2). $\mathrm{O}$ aumento nos teores foliares de K, a partir da aplicação de doses crescentes de $\mathrm{P}_{2} \mathrm{O}_{5}$, pode ser devido ao incremento da absorção ativa de K pelas raízes (Dutra et al., 1995), que é gerado, segundo Fernandes \& Souza (2006), pela hidrólise de ligações de alta energia, como de ATP ou PPi acumulados na planta em maior quantidade. Deve-se ressaltar, entretanto, o pequeno incremento no teor foliar de $\mathrm{P}$, mesmo que significativo, passando de $0,18 \mathrm{dag} \mathrm{kg}^{-1}$, na ausência de $\mathrm{N}$ e $\mathrm{P}_{2} \mathrm{O}_{5}$, para 0,21 dag kg-1, nas doses de 0,0 e $380 \mathrm{~kg} \mathrm{ha}^{-1}$ de $\mathrm{N}$ e 
Quadro 1. Comprimento (C), massa de matéria seca (MS), teores de N, P e K (NF, PF e KF) e relação K:N (KF:NF) das folhas D do abacaxi MD-2, à época da indução floral (11 meses após plantio), decorrentes da adubação com $\mathrm{N}, \mathrm{P}$ e K

\begin{tabular}{|c|c|c|c|c|c|c|c|c|c|}
\hline \multirow{2}{*}{ Tratamento } & \multicolumn{3}{|c|}{ Doses de nutrientes } & \multicolumn{6}{|c|}{ Características } \\
\hline & $\mathbf{N}$ & $\mathbf{P}_{2} \mathbf{O}_{5}$ & $\mathrm{~K}_{2} \mathrm{O}$ & $\mathbf{C}$ & MS & NF & PF & $\mathbf{K F}$ & $\mathrm{KF}: \mathrm{NF}$ \\
\hline & \multicolumn{3}{|c|}{$-\mathrm{kg} \mathrm{ha}^{-1}$} & $\mathrm{~cm}$ & g/folha & & $\operatorname{ag~kg}{ }^{-1}$ & & \\
\hline 1 & 300 & 120 & 300 & 69,30 & 5,81 & 1,54 & 0,18 & 3,41 & 2,21 \\
\hline 2 & 300 & 120 & 700 & 75,81 & 6,57 & 1,59 & 0,18 & 4,32 & 2,72 \\
\hline 3 & 300 & 280 & 300 & 76,39 & 6,57 & 1,54 & 0,20 & 3,59 & 2,33 \\
\hline 4 & 300 & 280 & 700 & 73,95 & 6,43 & 1,54 & 0,19 & 4,23 & 2,75 \\
\hline 5 & 700 & 120 & 300 & 75,03 & 6,77 & 1,68 & 0,18 & 3,32 & 1,98 \\
\hline 6 & 700 & 120 & 700 & 71,96 & 6,37 & 1,80 & 0,18 & 4,09 & 2,28 \\
\hline 7 & 700 & 280 & 300 & 75,99 & 6,63 & 1,73 & 0,20 & 3,52 & 2,04 \\
\hline 8 & 700 & 280 & 700 & 76,18 & 6,87 & 1,61 & 0,19 & 4,12 & 2,56 \\
\hline 9 & 50 & 120 & 300 & 66,11 & 5,36 & 1,63 & 0,19 & 4,13 & 2,53 \\
\hline 10 & 300 & 20 & 300 & 72,66 & 6,36 & 1,49 & 0,17 & 2,78 & 1,86 \\
\hline 11 & 300 & 120 & 50 & 69,68 & 5,71 & 1,63 & 0,18 & 4,02 & 2,46 \\
\hline 12 & 950 & 280 & 700 & 73,31 & 6,68 & 1,73 & 0,18 & 3,55 & 2,06 \\
\hline 13 & 700 & 380 & 700 & 73,89 & 6,17 & 1,73 & 0,19 & 4,14 & 2,40 \\
\hline 14 & 700 & 280 & 950 & 72,75 & 6,58 & 1,59 & 0,18 & 3,76 & 2,37 \\
\hline 15 & 500 & 200 & 500 & 74,65 & 6,61 & 1,56 & 0,19 & 3,81 & 2,44 \\
\hline 16 & 0 & 0 & 0 & 56,67 & 4,03 & 1,38 & 0,18 & 1,90 & 1,38 \\
\hline
\end{tabular}

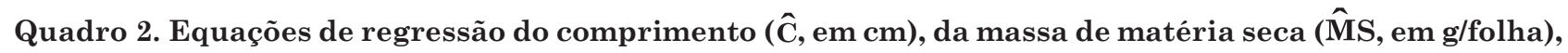

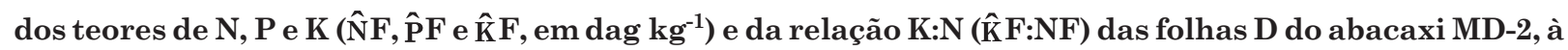
época da indução floral (11 meses após plantio), decorrentes da adubação com $\mathrm{N}, \mathrm{P}$ e K $\left(\mathrm{N}, \mathrm{P}_{2} \mathrm{O}_{5}\right.$ e $\left.\mathrm{K}_{2} \mathrm{O}\right)$

\begin{tabular}{|c|c|}
\hline Equação & $\mathbf{R}^{2}$ \\
\hline$\hat{\mathrm{C}}=58,46+0,0385 \mathrm{~N}+0,0258 \mathrm{~K}-0,000033^{* *} \mathrm{~N}^{2}-0,000024^{*} \mathrm{~K}^{2}$ & 0,85 \\
\hline$\hat{\mathrm{MS}}=4,30+0,0048 \mathrm{~N}+0,0035 \mathrm{~K}-0,0000038^{*} \mathrm{~N}^{2}-0,000003^{\circ} \mathrm{K}^{2}$ & 0,85 \\
\hline$\hat{\mathrm{NF}}=1,40+0,00027^{* *} \mathrm{~N}+0,00064 \mathrm{P}+0,00020 \mathrm{~K}-0,0000013^{\circ} \mathrm{PK}$ & 0,65 \\
\hline$\hat{\mathrm{PF}}=0,1803-0,00002^{* *} \mathrm{~N}+0,000074^{* *} \mathrm{P}$ & 0,71 \\
\hline$\hat{\mathrm{K}} \mathrm{F}=2,38+0,0113 \mathrm{P}+0,00074^{\circ} \mathrm{K}-0,000025^{* *} \mathrm{P}^{2}$ & 0,61 \\
\hline$\hat{\mathrm{K} F}: \mathrm{NF}=1,66-0,00083^{* *} \mathrm{~N}+0,00749 \mathrm{P}+0,00069^{*} \mathrm{~K}-0,000016{ }^{* *} \mathrm{P}^{2}$ & 0,76 \\
\hline
\end{tabular}

o, *,**: significativo a 10,5 e $1 \%$, respectivamente.

$\mathrm{P}_{2} \mathrm{O}_{5}$, respectivamente (Quadros 1 e 2). Da mesma forma, Spironello et al. (2004) observaram reduzido incremento na concentração foliar de $\mathrm{P}$, em plantas de abacaxi que receberam aplicação de doses crescentes de fertilizante fosfatado, em solos com baixos teores iniciais de $\mathrm{P}$.

$\mathrm{O} \mathrm{KCl}$, aplicado em doses crescentes, aumentou os teores foliares de $\mathrm{N}$ e $\mathrm{K}$ e a relação $\mathrm{K}: \mathrm{N}$ de forma linear, mas não interferiu nos teores de P (Quadros 1 e 2). Esses resultados são corroborados, em parte, pelos obtidos no trabalho de Spironello et al. (2004), que também não observaram efeito da aplicação de K sobre os teores foliares de P. Em contraponto, esses autores reportam que a aplicação de K reduziu marcadamente os teores foliares de $\mathrm{N}$ - exatamente o inverso do observado no presente estudo.

Os teores foliares máximos estimados (1,72, 0,21 e 4,36 dag kg-1 de N, P e K, respectivamente; Quadro 2) foram adequados, se comparados a valores de referência determinados por Reuter \& Robinson (1988) na fração aclorofilada da folha D. Siebeneichler et al. (2002) reportam que a parte aclorofilada da folha D apresenta maiores teores de $\mathrm{P}$ e de $\mathrm{K}$ do que outras partes da folha. Ainda assim, o teor foliar máximo de $\mathrm{K}$, estimado no presente estudo, foi $68 \%$ maior do que o apresentado por Siebeneichler et al. (2002), que encontraram 2,6 dag $\mathrm{kg}^{-1}$ de $\mathrm{K}$ na parte aclorofilada da folha D do abacaxi Smooth Cayene. 


\section{Produtividade, massa e diâmetro dos frutos}

A aplicação de $\mathrm{P}$, na forma de superfosfato triplo, não causou efeito significativo sobre quaisquer das variáveis de desenvolvimento do fruto avaliadas (Quadros 3 e 4), mesmo com o solo apresentando reduzido teor inicial desse nutriente $\left(2,56 \mathrm{mg} \mathrm{dm}^{-3}\right)$. Esse fato já é bem estabelecido e comprovado para a cultura do abacaxi, como reportam os trabalhos de Botrel et al. (1991) e Spironello et al. (2004). A reduzida ou nula resposta de produção do abacaxi à aplicação de $\mathrm{P}$ pode ser consequência de elevada capacidade de recuperação do $\mathrm{P}$ nativo do solo pelas raízes, provavelmente por efeito de elevada associação micorrízica e, ou, pela elevada acidificação na rizosfera da planta. Essa afirmação é consistente, pois, mesmo sem adição do nutriente, o teor estimado na parte aclorofilada, utilizando-se a dose de $\mathrm{N}$ para a produção máxima (Quadro 4), foi de $0,17 \mathrm{dag} \mathrm{kg}^{-1}$ de $\mathrm{P}$ (Quadro 2), valor compreendido na faixa de suficiência estipulada para a fração aclorofilada da folha $\mathrm{D}$ por Reuter \& Robinson (1988): 0,14 a 0,35 dag $\mathrm{kg}^{-1}$ de P.

Para todas as características de desenvolvimento dos frutos avaliadas houve efeito quadrático de doses crescentes de $\mathrm{N}$ e K (Quadros 3 e 4). Os máximos de produtividade $\left(65,4 \mathrm{t} \mathrm{ha}^{-1}\right)$ e de MCC (1.277 g) foram obtidos com a aplicação de 650,6 e $735,9 \mathrm{~kg} \mathrm{ha}^{-1}$, correspondendo a 12,7 e 14,4 g/planta de $\mathrm{N} \mathrm{e} \mathrm{K}_{2} \mathrm{O}$, respectivamente. Considerando que a aplicação de doses crescentes de $\mathrm{K}$ aumentou os teores de $\mathrm{K}$ no solo (KS) de forma linear, segundo a equação $\hat{K} S=11,8+$ $0,102919^{* *} \mathrm{~K} ; \mathrm{R}^{2}=0,93$ e coeficiente significativo a $1 \%$, a dose de $\mathrm{K}_{2} \mathrm{O}$ que proporcionou maior produtividade estava associada a um teor de $87,6 \mathrm{mg} \mathrm{dm}^{-3}$ de K no solo.
Spironello et al. (2004), trabalhando com a cultivar Smooth Cayene, obtiveram produtividade máxima de frutos com coroa de $72 \mathrm{t} \mathrm{ha}^{-1}$ aplicando $498 \mathrm{e}$ $384 \mathrm{~kg} \mathrm{ha}^{-1}$ de $\mathrm{N}$ e $\mathrm{K}_{2} \mathrm{O}$, respectivamente. Essas doses, a princípio, estão bem abaixo das doses máximas estimadas no presente estudo (650,6 e 735,9 $\mathrm{kg} \mathrm{ha}^{-1}$ de $\mathrm{Ne} \mathrm{K}_{2} \mathrm{O}$, respectivamente). Entretanto, Spironello et al. (2004) utilizaram densidade de plantio de 30.300 plantas ha-1, enquanto, no presente estudo, a densidade de plantio foi de 51.280 plantas $\mathrm{ha}^{-1}$. Nesse caso, ao calcular as doses por planta para o trabalho de Spironello et al. (2004), obtêm-se 16,4 e 12,7 g/planta de $\mathrm{Ne} \mathrm{K}_{2} \mathrm{O}$, respectivamente, ou seja, doses muito mais próximas das determinadas no presente estudo para a produtividade máxima (12,7 e 14,4 g/planta de $\mathrm{Ne} \mathrm{K}_{2} \mathrm{O}$, respectivamente). Assim, independentemente do espaçamento de plantio, para que as plantas dos dois cultivares, individualmente, expressassem sua máxima capacidade produtiva, seria necessária a aplicação de doses muito próximas de $\mathrm{Ne}_{2} \mathrm{O}$, por planta, fato que não é explicitado ao se recomendar a adubação por área. Contudo, apesar de mais próximas, a ordem de magnitude foi completamente inversa: enquanto a cultivar Smooth Cayene necessitou de maior dose de $\mathrm{N}$, em relação à cultivar MD-2, para atingir produtividade máxima, esta necessitou de maior dose de $\mathrm{K}_{2} \mathrm{O}$, em relação à cultivar Smooth Cayene.

Considerando os resultados apresentados, fica claro que a recomendação de doses de nutrientes por área, para a cultura do abacaxi, não é adequada, a não ser que se recomende, também, a densidade de plantio equivalente, ou seja, o arranjo de plantas que proporcione a melhor resposta em produtividade. Essa sugestão sustenta-se na forma como é realizada a adubação (axilar), dada a pequena extensão do sistema

Quadro 3. Produtividade (Prod), massa do fruto com (MCC) e sem coroa (MSC), diâmetro do fruto (DF) e percentagem da coroa em relação à $\mathrm{MCC}(\% \mathrm{MC})$, decorrentes da adubação com $\mathrm{N}, \mathrm{P}$ e $\mathrm{K}$

\begin{tabular}{|c|c|c|c|c|c|c|c|c|}
\hline \multirow{2}{*}{ Tratamento } & \multicolumn{3}{|c|}{ Doses de nutrientes } & \multicolumn{5}{|c|}{ Variáveis } \\
\hline & $\mathbf{N}$ & $\mathbf{P}_{2} \mathbf{O}_{5}$ & $\mathbf{K}_{2} \mathbf{O}$ & Prod & MCC & MSC & DF & $\% \mathbf{M C}$ \\
\hline & 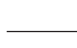 & $\mathrm{kg}$ ha & - & $\mathrm{tha}^{-1}$ & $\longrightarrow$ & - & $\mathrm{cm}$ & $\%$ \\
\hline 1 & 300 & 120 & 300 & 52,94 & $1.034,0$ & 767,8 & 10,72 & 25,74 \\
\hline 2 & 300 & 120 & 700 & 63,22 & $1.234,7$ & 954,4 & 11,31 & 22,71 \\
\hline 3 & 300 & 280 & 300 & 59,15 & $1.155,2$ & 882,9 & 10,98 & 23,57 \\
\hline 4 & 300 & 280 & 700 & 62,03 & $1.211,5$ & 946,0 & 11,23 & 21,91 \\
\hline 5 & 700 & 120 & 300 & 62,44 & $1.219,5$ & 963,3 & 11,08 & 21,01 \\
\hline 6 & 700 & 120 & 700 & 58,75 & $1.147,5$ & 904,2 & 10,89 & 21,20 \\
\hline 7 & 700 & 280 & 300 & 61,85 & $1.208,1$ & 925,8 & 11,13 & 23,36 \\
\hline 8 & 700 & 280 & 700 & 64,05 & $1.251,0$ & $1.002,5$ & 11,41 & 19,86 \\
\hline 9 & 50 & 120 & 300 & 51,73 & $1.010,4$ & 737,9 & 10,52 & 26,96 \\
\hline 10 & 300 & 20 & 300 & 59,79 & $1.167,7$ & 887,1 & 11,05 & 24,03 \\
\hline 11 & 300 & 120 & 50 & 56,93 & $1.111,9$ & 812,4 & 10,79 & 26,94 \\
\hline 12 & 950 & 280 & 700 & 64,93 & $1.268,1$ & 985,2 & 11,33 & 22,31 \\
\hline 13 & 700 & 380 & 700 & 66,25 & $1.294,0$ & $1.012,7$ & 11,37 & 21,74 \\
\hline 14 & 700 & 280 & 950 & 65,02 & $1.270,0$ & 995,5 & 11,39 & 21,61 \\
\hline 15 & 500 & 200 & 500 & 64,02 & $1.250,3$ & 976,9 & 11,24 & 21,86 \\
\hline 16 & 0 & 0 & 0 & 38,81 & 758,0 & 469,1 & 9,17 & 38,11 \\
\hline
\end{tabular}




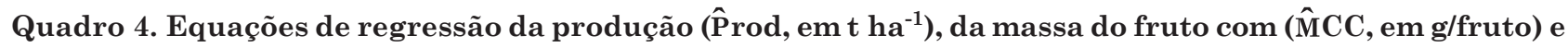
sem coroa ( $\hat{\mathrm{MSC}}$, em g), do diâmetro do fruto ( $(\hat{\mathrm{D} F}, \mathrm{em} \mathrm{cm})$ e da porcentagem da coroa em relação à MCC ( $\hat{\mathrm{C}})$, decorrentes da adubação com $\mathrm{N}, \mathrm{P}$ e K $\left(\mathrm{N}, \mathrm{P}_{2} \mathrm{O}_{5}\right.$ e $\left.\mathrm{K}_{2} \mathrm{O}\right)$

\begin{tabular}{clcc}
\hline Equação & $\mathbf{R}^{2}$ & N Máx. & K2O Máx. \\
\hline$\hat{P}$ rod $=42,02+0,0433 \mathrm{~N}+0,0250 \mathrm{~K}-0,000033^{* *} \mathrm{~N}^{2}-0,000017^{\circ} \mathrm{K}^{2}$ & 0,84 & 650,6 & $\mathrm{~kg} \mathrm{ha}^{-1}$ \\
$\hat{\mathrm{M} C C}=820,80+0,8462 \mathrm{~N}+0,4888 \mathrm{~K}-0,00065^{* *} \mathrm{~N}^{2}-0,00033^{\circ} \mathrm{K}^{2}$ & 0,84 & 650,6 & 735,9 \\
$\hat{\mathrm{MSC}}=524,88+0,8623 \mathrm{~N}+0,5755 \mathrm{~K}-0,00067^{* *} \mathrm{~N}^{2}-0,00040^{\circ} \mathrm{K}^{2}$ & 0,89 & 648,2 & 711,8 \\
$\hat{\mathrm{D}} \mathrm{F}=9,55+0,0034 \mathrm{~N}+0,0026 \mathrm{~K}-0,0000028^{* *} \mathrm{~N}^{2}-0,0000019^{* *} \mathrm{~K}^{2}$ & 0,84 & 594,0 & 691,5 \\
$\hat{\text { C }}=35,93-0,0267 \mathrm{~N}-0,0246 \mathrm{~K}+0,000022^{* *} \mathrm{~N}^{2}+0,000020^{* *} \mathrm{~K}^{2}$ & 0,92 & $609,3^{(1)}$ & $625,8^{(1)}$ \\
\hline
\end{tabular}

(1) Essas doses geraram a menor $\%$ MC. ${ }^{\circ},{ }^{*},{ }^{* *}$ : significativo a 10,5 e $1 \%$, respectivamente.

radicular das plantas de abacaxi, as quais, segundo Hanafi \& Halimah (2004), apresentam elevada relação parte aérea:raiz, sendo que o sistema radicular corresponde a, aproximadamente, 5 \% da matéria seca da planta. Portanto, uma maior densidade de plantio para o abacaxi não causaria maior taxa de recuperação dos nutrientes aplicados, como, segundo Pavan et al. (1994), tende a ocorrer para culturas com sistema radicular mais desenvolvido, o que mais uma vez reforça a recomendação de fertilizantes por planta e não por área.

A massa máxima do fruto sem coroa (MSC), o diâmetro máximo do fruto (DF) e a menor percentagem da coroa em relação à MCC (\% MC) foram estimados em 1.009,3 g, 11,5 cm e 20,3\%, respectivamente, com doses máximas de $\mathrm{N}$ e $\mathrm{K}_{2} \mathrm{O}$ um pouco menores do que as selecionadas para a produtividade e a MCC (Quadro 4).

O N e o K são os nutrientes responsáveis pelo maior crescimento do fruto de abacaxi (Quadro 4), como atestam os trabalhos de Bhugaloo (1998), Veloso et al. (2001), Razzaque \& Hanafi (2001) e Spironello et al. (2004). Entretanto, o efeito da adubação nitrogenada no crescimento do fruto foi mais pronunciado do que o efeito da adubação potássica, o que é evidenciado pelos coeficientes das equações de regressão selecionadas para as variáveis de desenvolvimento do fruto estudadas (Quadro 4), sendo esse resultado corroborado pelo trabalho de Spironello et al. (2004).

As correlações entre as características da folha D, no momento da indução floral, e as características de desenvolvimento do fruto revelam que o comprimento e a massa de matéria seca da folha D apresentaram maior relação com o desenvolvimento dos frutos do que os teores foliares de nutrientes (Quadro 5).

Utilizando-se as doses de $\mathrm{N}$ e $\mathrm{K}_{2} \mathrm{O}$ que proporcionaram maiores MCC e MSC (Quadro 4), foram estimados, como base nas equações do quadro 2 , o comprimento e a produção de matéria seca da folha
$\mathrm{D}$ que poderiam gerar frutos mais pesados, sendo: $75,5 \mathrm{~cm}$ e $6,77 \mathrm{~g}$ para a MCC e $75,8 \mathrm{~cm}$ e $6,79 \mathrm{~g}$ para a MSC, respectivamente. Esses valores estimados de comprimento da folha $\mathrm{D}$ confirmam, para o abacaxi MD-2, a afirmação de Reinhardt \& Cunha (2000) feita para o abacaxi Smooth Cayenne, ou seja, de que a indução floral artificial deve ser realizada quando o comprimento da folha $\mathrm{D}$ for maior do que $70 \mathrm{~cm}$, para que se obtenham frutos mais pesados. Nesse caso, van de Poel et al. (2009) consideram que plantas de abacaxi MD-2 cuja folha D apresente comprimento próximo a $74 \mathrm{~cm}$ estão completamente maduras, respondendo em $100 \%$ dos casos à indução floral artificial.

Quadro 5. Correlação do desenvolvimento, dos teores de nutrientes e razão dos nutrientes da folha $D$, no momento da indução floral, com características de produção dos frutos do abacaxi MD-2

\begin{tabular}{|c|c|c|c|c|}
\hline \multirow{2}{*}{$\begin{array}{l}\text { Característica } \\
\text { da folha } D^{(1)}\end{array}$} & \multicolumn{4}{|c|}{ Característica do fruto $^{(2)}$} \\
\hline & $\mathrm{MCC}$ & MSC & DF & $\% \mathbf{M C}$ \\
\hline & & & & \\
\hline Massa & $0,929^{* *}$ & $0,945^{* *}$ & $0,938^{* *}$ & $-0,953^{* *}$ \\
\hline Comprimento & $0,919^{* *}$ & $0,927^{* *}$ & $0,932^{* *}$ & $-0,924^{* *}$ \\
\hline $\mathrm{N}$ & $0,564^{*}$ & $0,578^{*}$ & $0,535^{*}$ & $-0,613^{*}$ \\
\hline $\mathrm{P}$ & $0,161^{\mathrm{ns}}$ & $0,152^{\mathrm{ns}}$ & $0,154^{\mathrm{ns}}$ & $-0,130 \mathrm{~ns}$ \\
\hline $\mathrm{K}$ & $0,639^{* *}$ & $0,652^{* *}$ & $0,701^{* *}$ & $-0,702^{* *}$ \\
\hline $\mathrm{K}: \mathrm{N}$ & $0,551^{*}$ & $0,560^{*}$ & $0,639^{* *}$ & $-0,607^{* *}$ \\
\hline $\mathrm{N}: \mathrm{K}$ & $-0,660^{* *}$ & $-0,669^{* *}$ & $-0,752^{* *}$ & $0,730^{* * *}$ \\
\hline $\mathrm{N}: \mathrm{P}$ & $0,429 \mathrm{~ns}$ & $0,448^{\mathrm{ns}}$ & $0,407 \mathrm{~ns}$ & $-0,496 \mathrm{~ns}$ \\
\hline $\mathrm{P}: \mathrm{N}$ & $-0,466^{\mathrm{ns}}$ & $-0,482 \mathrm{~ns}$ & $-0,451^{\mathrm{ns}}$ & $0,526^{*}$ \\
\hline $\mathrm{K}: \mathrm{P}$ & $0,653^{* *}$ & $0,669^{* *}$ & $0,722^{* *}$ & $-0,731^{* *}$ \\
\hline $\mathrm{P}: \mathrm{K}$ & $-0,748^{* *}$ & $-0,762^{* *}$ & $-0,825^{* *}$ & $0,831^{* *}$ \\
\hline
\end{tabular}

(1) $\mathrm{N}, \mathrm{P}, \mathrm{K}$ e $\mathrm{Y}_{\mathrm{i}}: \mathrm{X}_{\mathrm{i}}$ = teores de nitrogênio, fósforo, potássio e razão entre esses nutrientes, na parte basal aclorofilada da folha D. ${ }^{(2)} \mathrm{MCC}, \mathrm{MSC}, \mathrm{DF}$ e \% MC = massa dos frutos com e sem coroa, diâmetro do fruto e percentagem da coroa em relação a MCC; ${ }^{\text {ns }}$, ${ }^{*} \mathrm{e}^{* *}$, não significativo e significativo a 5 e $1 \%$, respectivamente. 
Os teores foliares de $\mathrm{N}$ e K mostraram correlação com o desenvolvimento do fruto, enquanto o teor de $\mathrm{P}$ não apresentou correlação significativa com qualquer das características de desenvolvimento de fruto estudadas (Quadro 5).

Considerando as doses de $\mathrm{N}$ e $\mathrm{K}_{2} \mathrm{O}$ que proporcionaram maior massa de frutos com (MCC) e sem coroa (MSC) (Quadro 4), estimou-se, por meio das equações de absorção (Quadro 2), que elas estavam associadas aos seguintes teores foliares de $\mathrm{N}$ e K: 1,72 e 2,91 dag kg-1 tanto para a MCC quanto para a MSC. Nesse caso, utilizou-se para o cálculo a dose 0 (zero) de $\mathrm{P}_{2} \mathrm{O}_{5}$, visto que o $\mathrm{P}$ não gerou qualquer efeito sobre a massa dos frutos.

As razões $\mathrm{K}: \mathrm{N}$ e $\mathrm{K}: \mathrm{P}$, na parte basal aclorofilada da folha D, apresentaram correlação positiva com a MCC, a MSC e o diâmetro de fruto, e correlação negativa com a \% MC, enquanto as razões $\mathrm{N}: \mathrm{K}$ e P:K apresentaram efeito inverso (Quadro 5), como seria de se esperar. Entretanto, o benefício de acréscimo no teor foliar de $\mathrm{K}$, em relação aos teores de $\mathrm{N}$ e $\mathrm{P}$, foi menor do que o prejuízo no desenvolvimento do fruto causado pelo acréscimo nos teores foliares de $\mathrm{N}$ e $\mathrm{P}$, em relação ao teor de $\mathrm{K}$, especialmente para a razão P:K (Quadro 5).

\section{Qualidade dos frutos}

Em contraponto à ausência de efeito sobre características de desenvolvimento dos frutos, a aplicação de $\mathrm{P}$ em doses crescentes proporcionou efeito linear positivo sobre os teores de sólidos solúveis totais (SST) e quadrático sobre a acidez total titulável (ATT) e sobre a razão entre essas variáveis (ratio), sendo os mesmos efeitos observados para a adição de $\mathrm{K}$ (Quadros 6 e 7). O efeito positivo da adubação potássica sobre a qualidade dos frutos de abacaxi já é bem estabelecido na literatura (Spironello et al., 2004). Contudo, para a adubação fosfatada, geralmente não se encontra, na literatura, qualquer efeito sobre a qualidade dos frutos de abacaxi (Botrel et al., 1991; Spironello et al., 2004).

O efeito da adubação potássica sobre a qualidade dos frutos foi, entretanto, muito superior ao da adubação fosfatada, o que é revelado pelos coeficientes das equações de regressão (Quadros 6 e 7). As doses máximas de $\mathrm{P}_{2} \mathrm{O}_{5}$ e $\mathrm{K}_{2} \mathrm{O}$ foram estimadas em 205,8 e $703,4 \mathrm{~kg} \mathrm{ha}{ }^{-1}$, respectivamente, para a máxima ATT, sendo esta igual a $0,47 \%$ de ácido cítrico. Considerando que o teor de $\mathrm{P}$ no solo (PS) aumentou de forma linear, quando foram aplicadas doses crescentes de $\mathrm{P}_{2} \mathrm{O}_{5}$, segundo a equação $\hat{\mathrm{p}}=0,339+0,0899^{* *} \mathrm{P} ; \mathrm{R}^{2}=0,89 \mathrm{e}$ coeficiente significativo a $1 \%$, a dose de $\mathrm{P}_{2} \mathrm{O}_{5}$ que proporcionou maior ATT estava associada a um teor de $18,8 \mathrm{mg} \mathrm{dm}^{-3}$ de $\mathrm{P}$ no solo. Nesse caso, o teor de acidez na polpa do abacaxi é fundamental para que este seja bem aceito no mercado consumidor, pois, segundo Bugaloo (1998), a baixa acidez da polpa proporciona fruto com pouco aroma e de gosto insípido. Para esse autor, quando a acidez é muito elevada, há redução na sensação de doçura, gerando uma fruta com sabor desagradável.

Com as maiores doses de $\mathrm{P}$ e $\mathrm{K}$ utilizadas no espaço experimental, o teor de SST máximo foi estimado em 18,2 ${ }^{\circ}$ Brix. Para Morgan \& Thompson (2000), o abacaxi MD-2 pode alcançar valores de SST entre 15 e $17^{\circ}$ Brix, bem próximo do encontrado no presente trabalho. Por outro lado, Rebolledo et al. (2006)

Quadro 6. Sólidos solúveis totais (SST), acidez total titulável (ATT) e ratio (R) na polpa do fruto do abacaxi MD-2, decorrentes da adubação com N, P e K

\begin{tabular}{|c|c|c|c|c|c|c|}
\hline \multirow{2}{*}{ Tratamento } & \multicolumn{3}{|c|}{ Nutrientes } & \multicolumn{3}{|c|}{ Variáveis } \\
\hline & $\mathbf{N}$ & $\mathbf{P}_{2} \mathbf{O}_{5}$ & $\mathbf{K}_{2} \mathbf{O}$ & SST & ATT & $\mathbf{R}$ \\
\hline & \multicolumn{3}{|c|}{$\mathrm{kg} \mathrm{ha} \mathrm{H}^{-1}$} & ${ }^{\circ}$ Brix & \% de Ác. Cítrico & \\
\hline 1 & 300 & 120 & 300 & 15,33 & 0,40 & 38,03 \\
\hline 2 & 300 & 120 & 700 & 16,14 & 0,43 & 37,45 \\
\hline 3 & 300 & 280 & 300 & 15,62 & 0,35 & 44,64 \\
\hline 4 & 300 & 280 & 700 & 16,25 & 0,41 & 39,47 \\
\hline 5 & 700 & 120 & 300 & 14,90 & 0,31 & 47,51 \\
\hline 6 & 700 & 120 & 700 & 15,00 & 0,39 & 38,41 \\
\hline 7 & 700 & 280 & 300 & 14,94 & 0,35 & 42,70 \\
\hline 8 & 700 & 280 & 700 & 15,20 & 0,41 & 37,10 \\
\hline 9 & 50 & 120 & 300 & 15,68 & 0,40 & 39,31 \\
\hline 10 & 300 & 20 & 300 & 15,36 & 0,34 & 45,57 \\
\hline 11 & 300 & 120 & 50 & 14,93 & 0,28 & 53,01 \\
\hline 12 & 950 & 280 & 700 & 14,74 & 0,33 & 44,02 \\
\hline 13 & 700 & 380 & 700 & 15,24 & 0,37 & 41,06 \\
\hline 14 & 700 & 280 & 950 & 15,56 & 0,38 & 40,75 \\
\hline 15 & 500 & 200 & 500 & 15,15 & 0,39 & 38,37 \\
\hline 16 & 0 & 0 & 0 & 13,80 & 0,20 & 68,82 \\
\hline
\end{tabular}


Quadro 7. Equações de regressão de sólidos solúveis totais ( $\widehat{\mathrm{S}} \mathrm{ST}$, em ${ }^{\circ} \mathrm{Brix}$ ), da acidez total titulável (ÂTT, em \% de ácido cítrico) e do ratio ( $\hat{\mathrm{R}})$ na polpa do fruto do abacaxi MD-2, decorrentes da adubação com $\mathrm{N}, \mathrm{P}$ e K $\left(\mathrm{N}, \mathrm{P}_{2} \mathrm{O}_{5}\right.$ e $\left.\mathrm{K}_{2} \mathrm{O}\right)$

\begin{tabular}{lc}
\hline \multicolumn{1}{c}{ Equação } & $\mathbf{R}^{2}$ \\
\hline$\hat{\mathrm{S} S T}=14,37+0,00142^{* * *} \mathrm{P}+0,00349^{* *} \mathrm{~K}-0,000004^{* *} \mathrm{NK}$ & 0,86 \\
$\hat{\mathrm{A} T T}=0,2213-0,00010^{*} \mathrm{~N}+0,000616 \mathrm{P}+0,000531 \mathrm{~K}-0,0000015^{*} \mathrm{P}^{2}-0,00000038^{* *} \mathrm{~K}^{2}$ & 0,90 \\
$\hat{\mathrm{R}}=64,87-0,0781 \mathrm{P}-0,0678 \mathrm{~K}+0,000202^{\circ} \mathrm{P}^{2}+0,0000543^{* *} \mathrm{~K}^{2}$ & 0,85 \\
\hline
\end{tabular}

${ }^{\circ}, *, * *$ : significativo a 10,5 e $1 \%$, respectivamente.

observaram valores de 11,9 ${ }^{\circ}$ Brix para os SST e de 0,35\% de ácido cítrico para a ATT, em trabalho realizado no México com o abacaxi MD-2.

A discrepância de resultados entre o presente trabalho (18,2 ${ }^{\circ}$ Brix e 0,47\% de ácido cítrico) e o trabalho de Rebolledo et al. (2006) pode ser explicada pela massa dos frutos alcançada em cada um deles. Enquanto neste estudo a massa de frutos máxima foi estimada em 1,28 kg, no trabalho de Rebolledo et al. (2006) ela foi de 1,83 kg, para uma densidade de plantio equivalente. Essa diferença na massa de frutos é determinante para os resultados de qualidade de polpa, uma vez que, segundo Reinhardt et al. (2004), frutos menores apresentam maiores teores de SST e ATT características positivas para o seu uso na indústria de sucos e também para o consumo do fruto in natura.

Smith (1988) reporta, nesse sentido, que frutos de abacaxi destinados ao consumo in natura devem apresentar teores de SST iguais ou superiores a 14 Brix. É interessante notar que esse valor é inferior ao encontrado no presente trabalho (18,2 ${ }^{\circ}$ Brix) e superior ao encontrado no trabalho de Rebolledo et al. (2006) (11,9 ${ }^{\circ}$ Brix). Assim, frutos mais pesados podem ter a sua qualidade comprometida, sendo inadequados para o consumo in natura, pois tendem a apresentar baixa aceitação pelo mercado consumidor.

A qualidade da fruta tem clara relação com as adubações potássica e nitrogenada. Enquanto a aplicação de ambos os nutrientes promoveu maior crescimento do fruto (Quadros 3 e 4), apenas a aplicação de $\mathrm{K}$ proporcionou melhora em sua qualidade, uma vez que a adição de doses crescentes de $\mathrm{N}$ causou efeito linear negativo sobre a ATT e interação negativa com a aplicação de K sobre o teor de SST (Quadros 6 e 7).

O efeito negativo da adubação nitrogenada na qualidade do fruto de abacaxi é reportado em diversos trabalhos (Bugaloo, 1998; Teixeira et al., 2002; Spironello et al., 2004). Nesse caso, segundo Spironello et al. (2004), o efeito negativo do N nos teores de SST e de ATT pode ser compensado pela aplicação de K, dado o efeito positivo desse nutriente nessas características de qualidade do fruto.
Esse fato é confirmado pelas correlações significativas e de elevadas magnitudes das relações foliares $\mathrm{K}: \mathrm{N}$ e $\mathrm{N}: \mathrm{K}$ com as características de qualidade dos frutos (Quadro 8). Essas relações foliares apresentaram maior correlação com as características de qualidade dos frutos do que o teor foliar isolado de $\mathrm{K}$ ou de $\mathrm{N}$, o qual não apresentou correlação significativa (Quadro 8). Vale ressaltar que as correlações das características de qualidade com a relação foliar K:N foram positivas e com a relação $\mathrm{N}: \mathrm{K}$, negativas, como seria de se esperar (Quadro 8). Esse resultado é confirmado por Martin-Prével (1961), que observou melhora na qualidade dos frutos (coloração, aroma, sabor e resistência ao transporte) à medida que a relação foliar $\mathrm{K}: \mathrm{N}$ se aproximava de $3: 1$, indicando que eventuais aumentos no teor foliar de $\mathrm{N}$, sem o correspondente incremento de K, poderiam alterar a qualidade da produção.

Quadro 8. Correlação do desenvolvimento, dos teores de nutrientes e razão dos nutrientes da folha $\mathrm{D}$, no momento da indução floral, com características de qualidade dos frutos do abacaxi MD-2

\begin{tabular}{lccc}
\hline \multirow{2}{*}{$\begin{array}{c}\text { Caracteristica } \\
\text { da folha } \mathbf{D}^{(1)}\end{array}$} & \multicolumn{2}{c}{ Característica do fruto } \\
\cline { 2 - 4 } & Acidez & SST & Ratio \\
\cline { 2 - 4 } & & $\mathrm{r}$ & \\
\cline { 2 - 4 } Massa & $0,610^{*}$ & $0,492^{\mathrm{ns}}$ & $-0,726^{* *}$ \\
Comprimento & $0,618^{*}$ & $0,555^{*}$ & $-0,720^{* *}$ \\
$\mathrm{~N}$ & $0,311^{\mathrm{ns}}$ & $0,038^{\mathrm{ns}}$ & $-0,471^{\mathrm{ns}}$ \\
$\mathrm{P}$ & $0,212^{\mathrm{ns}}$ & $0,321^{\mathrm{ns}}$ & $-0,180^{\mathrm{ns}}$ \\
$\mathrm{K}$ & $0,777^{* *}$ & $0,694^{* *}$ & $-0,782^{* *}$ \\
$\mathrm{~K}: \mathrm{N}$ & $0,802^{* *}$ & $0,820^{* *}$ & $-0,750^{* *}$ \\
$\mathrm{~N}: \mathrm{K}$ & $-0,832^{* *}$ & $-0,809^{* *}$ & $0,832^{* *}$ \\
$\mathrm{~N}: \mathrm{P}$ & $0,168^{\mathrm{ns}}$ & $-0,139^{\mathrm{ns}}$ & $-0,334^{\mathrm{ns}}$ \\
$\mathrm{P}: \mathrm{N}$ & $-0,207^{\mathrm{ns}}$ & $0,098^{\mathrm{ns}}$ & $0,374^{\mathrm{ns}}$ \\
$\mathrm{K}: \mathrm{P}$ & $0,785^{* *}$ & $0,664^{* *}$ & $-0,802^{* *}$ \\
$\mathrm{P}: \mathrm{K}$ & $-0,801^{* *}$ & $-0,691^{* *}$ & $0,862^{* *}$ \\
\hline
\end{tabular}

(1) $\mathrm{N}, \mathrm{P}, \mathrm{K}$ e $\mathrm{Y}_{\mathrm{i}}: \mathrm{X}_{\mathrm{i}}=$ teores de nitrogênio, fósforo, potássio e razão entre esses nutrientes, na parte basal aclorofilada da folha D. ${ }^{n s},{ }^{*} \mathrm{e}^{* *}$ : não significativo e significativo a 5 e $1 \%$, respectivamente. 
A máxima MCC estimada neste estudo foi de $1,28 \mathrm{~kg}$, obtida com as doses de $650,6,0,0$ e $735,9 \mathrm{~kg} \mathrm{ha}^{-1}$ de $\mathrm{N}, \mathrm{P}_{2} \mathrm{O}_{5}$ e $\mathrm{K}_{2} \mathrm{O}$, respectivamente, o que remete a uma relação $\mathrm{K}: \mathrm{N}$ de 1,63:1 (Quadro 2). Considerando a proposição de Martin-Prével (1961) como correta, o maior fruto produzido no presente estudo não seria o mais saboroso. Tomando-se as doses de $\mathrm{N}, \mathrm{P}_{2} \mathrm{O}_{5}$ e de $\mathrm{K}_{2} \mathrm{O}$ que proporcionaram a maior ATT $\left(0,0,205,8\right.$ e 703,4 $\mathrm{kg} \mathrm{ha}^{-1}$ de N, $\mathrm{P}_{2} \mathrm{O}_{5}$ e $\left.\mathrm{K}_{2} \mathrm{O}\right)$, estimou-se uma relação foliar K:N de 3,0:1 (Quadro 2), exatamente a recomendada por Martin-Prével (1961). Calculando-se a massa do fruto, o teor de SST e o ratio, a partir dessa relação $\left(0,0,205,8\right.$ e $703,4 \mathrm{~kg}^{\mathrm{h}} \mathrm{a}^{-1}$ de $\mathrm{N}, \mathrm{P}_{2} \mathrm{O}_{5}$ e $\mathrm{K}_{2} \mathrm{O}$, respectivamente), estimou-se que o fruto deveria apresentar MCC de $1001 \mathrm{~g}$, com correspondentes ATT de $0,47 \%$ de ácido cítrico, SST de $17,1^{\circ}$ Brix e ratio de 36,4 , para que atingisse a máxima qualidade. Pelo exposto, fica clara a dualidade de efeito do N: se por um lado esse nutriente aumenta a produtividade do abacaxi, por outro, reduz a qualidade do fruto. As exigências do mercado consumidor irão definir, nesse caso, a magnitude da dose de $\mathrm{N}$ a ser aplicada para o abacaxi.

\section{CONCLUSÕES}

1. A aplicação de doses crescentes de $\mathrm{N}$ e K promoveu acréscimo no desenvolvimento vegetativo, no desenvolvimento do fruto e na produtividade do abacaxi MD-2. Em contrapartida, a adição de $\mathrm{P}$ não causou qualquer efeito sobre as características de crescimento.

2. As máximas produtividade e massa de fruto foram alcançadas com a adição de 650,6 de $\mathrm{N}$ e $735,9 \mathrm{~kg} \mathrm{ha}^{-1} \mathrm{de}_{2} \mathrm{O}$, correspondendo a 12,7 e $14,4 \mathrm{~g} /$ planta, respectivamente.

3. Frutos mais pesados foram obtidos quando a folha $\mathrm{D}$ atingiu, no momento da indução floral, comprimento mínimo de $75,5 \mathrm{~cm}$ e apresentou teores foliares de $\mathrm{N}$ e K de 1,72 e 2,92 dag $\mathrm{kg}^{-1}$, respectivamente.

4. As características de qualidade do fruto foram reduzidas com aplicação de $\mathrm{N}$ e incrementadas com a adição de $\mathrm{P}$ e $\mathrm{K}$, sendo que as doses máximas de $205,8 \mathrm{~kg} \mathrm{ha}^{-1}$ de $\mathrm{P}_{2} \mathrm{O}_{5}$ e $703,4 \mathrm{~kg} \mathrm{ha}^{-1}$ de $\mathrm{K}_{2} \mathrm{O}$ corresponderam a 4,01 e 13,7 g/planta, respectivamente.

\section{LITERATURA CITADA}

BHUGALOO, R.A. Effect of different levels of nitrogen on yield and quality of pineapple variety Queen Victoria. Réduit, Mauritius, Food and Agricultural Research Council, 1998. p.75-79. (Technical Bulletin)

BOTREL, N.; SIQUEIRA, D.L.; PEDROSO NETO, J.C. \& PAULA, M.B. Efeito de diferentes fontes, níveis e modos de aplicação de fósforo na cultura do abacaxizeiro. Pesq. Agropec. Bras., 26:907-912, 1991.
CHAN, Y.K.; COOPENS, E.G. \& SANEWSKI, G.M. Breeding and variety improvement. In: BARTHOLOMEW, D.P.; PAULL, R.E. \& ROHRBACH, K.G., eds. The pineapple, botany, production and uses. Manoa, Honolulu, University of Hawaii, 2002. p.36-39.

DUTRA, L.F.; TAVARES, S.W.; SARTORETTO, L.M. \& VAHL, L.C. Resposta do feijoeiro ao fósforo em dois níveis de umidade no solo. R. Bras. Agroci., 1:91-96, 1995.

FERNANDES, M.S. \& SOUZA, S.R. Absorção de nutrientes. In: FERNANDES, M.S., ed. Nutrição mineral de plantas. Viçosa, MG, Sociedade Brasileira de Ciência do Solo, 2006. p.115-152.

HANAFI, M.M. \& HALIMAH, A. Nutrient supply and drymatter partitioning of pineapple cv. Josapine on sandy tin tailings. Fruits, 59:359-366, 2004.

LONGO, R.M. \& MELO, W.J. Hidrólise da uréia em Latossolos: Efeito da concentração de uréia, temperatura, $\mathrm{pH}$, armazenamento e tempo de incubação. R. Bras. Ci. Solo, 29:651-657, 2005.

MARTIN-PRÉVEL, P. Potassium, calcium et magnésium dans la nutrition de l'ananas em Guinée. III. Influence sur la qualité du fruit. Fruits, 16:161-80, 1961.

MORGAN, T. \& THOMPSON, T. Del Monte mixes and matches Costa Rican products. Americafruit, 3:45-47, 2000.

NIGHTINGALE, G.T. Potassium and phosphate nutrition of pineapple in relation to nitrate and carbohydrate reserves. Bot. Gazette, 104:191-223, 1942.

PAVAN, M.A.; CHAVES, J.C.D. \& ANDROCIOLI FILHO, A. Produção de café em função da densidade de plantio, adubação e tratamento fitossanitário. Turrialba, 44:227$231,1994$.

RAZZAQUE, A.H.M. \& HANAFI, M.M. Effect of potassium on growth, yield and quality of pineapple in tropical peat. Fruits, 56:45-49, 2001.

REBOLLEDO, A.M.; DEL ÁNGEL, A.L.P.; REBOLLEDO, L.M.; BECERRIL, A.E.R. \& URIZA, D.A. Rendimiento y calidad de fruto de cultivares de piña en densidades de plantación. R. Fitotec. Mex., 29:55-62, 2006.

REINHARDT, D.H. \& CUNHA, G.A.P. Manejo da floração. In: REINHARDT, D.H.; SOUZA, L.F.S. \& CABRAL, J.R.S. Abacaxi: Produção-aspectos técnicos. Brasília, Embrapa, 2000. p.41-45.

REINHARDT, D.H.; CABRAL, J.R.S.; SOUZA, L.F.S.; SANCHES, N.F. \& MATOS, A.P. Pérola and Smooth Cayene pineapple cultivars in the state of Bahia, Brazil: Growth, flowering, pests and diseases, yeld and fruit quality aspects. Fruits, 57:43-53, 2002.

REINHARDT, D.H.; MEDINA, V.M.; CALDAS, R.C.; CUNHA, G.A.P. \& ESTEVAM, R.F.H. Gradientes de qualidade em abacaxi 'Pérola' em função do tamanho e do estádio de maturação do fruto. R. Bras. Frutic., 26:544-546, 2004.

REUTER, D.J. \& ROBINSON, J.B. Plant analysis: An interpretation manual. Melbourne, Inkata, 1988. 218p. 
ROSOLEM, C.A.; FOLONI, J.S.S. \& OLIVEIRA, R.H. Dinâmica do nitrogênio no solo em razão da calagem e da adubação nitrogenada, com palha na superfície. Pesq. Agropec. Bras., 38:301-309, 2003.

SANTANA, L.L.A.; REINHARDT, D.H.; CUNHA, G.A.P. \& CALDAS, R.C. Altas densidades de plantio na cultura do abacaxi CV. Smooth Cayenne, sob condições de sequeiro. R. Bras. Frutic., 23:353-358, 2001.

SIEBENEICHLER, S.C.; MONNERAT, P.H.; CARVALHO, A.J.C. \& SILVA, J.A. Composição mineral da folha em abacaxizeiro: Efeito da parte da folha analisada. R. Bras. Frutic., 24:194-198, 2002.

SMITH, L.G. Indices of physiological maturity and eating quality in Smooth Cayenne pineapples. 2. Indices of eating quality. Queensland. J. Agric. An. Sci., 45:219-228, 1988.

SPIRONELLO, A.; QUAGGIO, J.A.; TEIXEIRA, L.A.J.; FURLANI, P.R. \& SIGRIST, J.M.M. Pineapple yeld and fruit quality effected by NPK fertilization in a tropical soil. R. Bras. Frutic., 26:155-159, 2004.
TEIXEIRA, L.A.J.; SPIRONELLO, A.; FURLANI, P.R. \& SIGRIST, J.M.M. Parcelamento da adubação NPK em abacaxizeiro. R. Bras. Frutic., 24:219-224, 2002.

van de POEL, B.; CEUSTERS, J. \& De PROFT, M.P. MD-2 pineapple plant maturity and flowering. Pineapple News, 16:10-12, 2009. Disponível em: <http://www.ishshorticulture.org/workinggroups/pineapple/ PineNews16.pdf> Acesso em: 7 de jul. 2010.

VELOSO, C.A.C.; OEIRAS, A.H.L.; CARVALHO, E.J.M. \& SOUZA, F.R.S. Resposta do abacaxizeiro à adição de nitrogênio, potássio e calcário em Latossolo Amarelo do nordeste Paraense. R. Bras. Frutic., 23:396-402, 2001. 